\title{
MEMÓRIA IMPLÍCITA E SUA CONTRIBUIÇÃO À REABILITAÇÃO DE UM PACIENTE AMNÉSTICO
}

\author{
RELATO DE CASO
}

\author{
SILVIA A. PRADO BOLOGNANI*, PAULA A. R. GOUVEIA*, \\ SONIA M. D. BRUCKI**, ORLANDO F. A. BUENO***-
}

\begin{abstract}
RESUMO - Mesmo pacientes severamente amnésticos são capazes de aprender informações novas, apoiados em estratégias de memória implícita. No presente estudo descrevemos uma experiência de reabilitação neuropsicológica, em que um paciente amnéstico, portador de lesão cerebral por anóxia, foi submetido a treino para uso de computador, visando sua capacitação semi-profissional. O treinamento teve duração de 14 semanas, e as técnicas de ensino utilizadas foram a prática repetitiva e a aprendizagem sem erros. O paciente obteve sucesso na realização das tarefas propostas, aprendendo a operar um editor de textos e mantendo o aprendizado após a passagem do tempo. Apesar disso, lembrava-se apenas vagamente da situação de aprendizagem. O presente estudo corrobora trabalhos anteriores que observam a interação entre mecanismos de memória implícita e resíduos de memória explícita operando na aquisição de conhecimentos em pacientes amnésticos. A aprendizagem de conhecimentos específicos vem se mostrando eficiente para a reabilitação de pacientes que sofreram lesões cerebrais.
\end{abstract}

PALAVRAS-CHAVE: memória implícita, reabilitação, cognição, amnésia, anóxia cerebral, aprendizagem de conhecimentos específicos.

\section{Contributions of implicit memory strategies to the rehabilitation of an amnesic patient: case report}

ABSTRACT - Even severe amnesic patients usually are able to learn new information using implicit memory strategies. In the present study we describe our experience in the rehabilitation of an amnesic patient who had suffered cerebral anoxia. He was taught to use a computer text editor, enabling him to sheltered work. The training program lasted 14 weeks and was based on errorless learning and drilling practice techniques. The patient succeeded in all tasks, even though he could not remember accurately the training sessions. These results meet previous studies which point out that interaction between implicit and residual explicit memory can provide a basis new learning in amnesic patients. The domain-specific knowledge acquisition is demonstrating effectiveness in neuropsychological rehabilitation of brain injured patients. knowledge.

KEY WORDS: implicit memory, rehabilitation, cognition, amnesia, cerebral anoxia, domain-specific

Desde a metade do século passado existem estudos sistemáticos procurando a descrição clínica e a compreensão científica dos problemas de memória, levando os teóricos a fazerem distinções conceituais, tais como a de memória de curto prazo e de longo prazo ${ }^{1}$. A partir da década de 70 , com

Universidade Federal de São Paulo / Escola Paulista de Medicina (UNIFESP/EPM), Departamento de Psicobiologia - Centro Paulista de Neuropsicologia (CPN): *Psicóloga do CPN; **Doutora em Neurologia pela UNIFESP e neurologista do CPN; ***Professor Adjunto do Departamento de Psicobiologia da UNIFESP e Coordenador Geral do CPN. Apoio Financeiro: Associação Fundo de Incentivo à Psicofarmacologia. Aceite: 26-junho-2000.

Dr. Orlando A. F. Bueno - Departamento de Psicobiologia EPM/UNIFESP - Rua Botucatu $862 / 1^{\circ}$ andar 04023-002 São Paulo SP - Brasil. 
os estudos com pacientes amnésticos ${ }^{2}$, elaborou-se a distinção entre os sistemas de memória explícita e memória implícita (ou memória declarativa e não declarativa, segundo outra terminologia).

A memória explícita é a lembrança consciente e voluntária de uma informação ou evento, que pode ser declarada, isto é, trazida à mente como uma proposição ou uma imagem. Os pacientes amnésticos apresentam prejuízo neste tipo de memória ${ }^{3}$.

A memória implícita é a capacidade de adquirir habilidades percepto-motoras ou cognitivas, através da exposição repetida a um estímulo ou atividade; estas experiências só podem ser aferidas pela melhora no desempenho do indivíduo, já que não são expressas de maneira consciente ou intencional ${ }^{2,4}$. Os pacientes amnésticos apresentam estas capacidades preservadas, o que pode ser observado através do efeito de pré-ativação (priming), de condicionamento, e de aquisição de habilidades ${ }^{3}$.

Assim, mesmo os pacientes com graves prejuízos de memória explícita preservam, em algum grau, a capacidade de aprendizagem. O comportamento e o desempenho destas pessoas mostram-se influenciados por suas experiências recentes, apesar de sua incapacidade de recordar-se ou mesmo de ter consciência de tais experiências ${ }^{2,4-6}$.

Wilson, Baddeley e Cockburn ${ }^{7}$ observaram que através da prática repetitiva e utilizando mecanismos de memória implícita, pacientes amnésticos podem ser treinados em tarefas complexas, como, por exemplo, utilizar uma agenda eletrônica. Glisky ${ }^{8}$ relata o caso de um paciente severamente amnéstico que foi capaz de operar um programa de cadastramento de dados em computador, aprendendo a identificar as informações que deveriam ser cadastradas.

Baddeley ${ }^{9}$, entretanto, ressalta que embora o treinamento de tarefas complexas seja possível, existem problemas específicos da aprendizagem apoiada em memória implícita, os quais dificultam o seu uso em reabilitação. Entre estes, destacam-se a persistência dos erros, isto é, a incapacidade dos pacientes de monitorar e corrigir o seu desempenho, e a hiperespecificidade do conhecimento, ou a incapacidade de generalizar o conhecimento adquirido a outros contextos.

Assim, um desafio aos profissionais que trabalham com reabilitação neuropsicológica é utilizar os modelos experimentais e teóricos de sistemas de memória para melhorar o desempenho de pacientes amnésticos em seu dia-a-dia. Conforme apontam Glisky e Schacter ${ }^{6}$, é necessário que os estudos em reabilitação possibilitem: a) avaliar a quantidade e complexidade das informações que podem ser aprendidas por pacientes com problemas de memória, e b) investigar se é possível aproveitar as capacidades de aprendizagem preservadas para ensinar-lhes novos conhecimentos e habilidades que sejam úteis em suas vidas diárias. Nesta linha de trabalho destacam-se os estudos que investigaram métodos de aprendizagem mais eficientes para pacientes amnésticos ${ }^{7,10}$, além da série de trabalhos nos quais os pacientes foram ensinados a operar computadores, aprendendo tarefas que pudessem ser usadas em ambientes profissionais ${ }^{6,8,11}$.

O objetivo deste estudo é descrever o procedimento de reabilitação neuropsicológica em um caso clínico, verificando se um treinamento apoiado em funções cognitivas preservadas pode auxiliar um paciente amnéstico a adquirir habilidades que tenham utilidade prática e imediata em sua vida.

\section{MÉTODO}

\section{Caso}

FS, 23 anos, masculino, estudante de nível superior, submeteu-se a septoplastia nasal 2 anos antes da admissão para reabilitação, e durante esta cirurgia teve parada cárdio-respiratória e anóxia cerebral. As queixas na admissão eram: dificuldades para lembrar-se de conversas, do nome de pessoas que viesse a conhecer e de fatos ocorridos recentemente; repetições seguidas de perguntas e de assuntos (percebidas pela família e às vezes por ele mesmo); dificuldade de compreensão de textos, não conseguindo, por isso, retomar os estudos; atitudes ingênuas, como por exemplo, não entender comentários maldosos. Ele teve uma grande redução no seu círculo de amizades e ficava envergonhado frente às garotas devido ao seu problema de memória. 
Ao exame neurológico não apresentava déficits motores ou sensitivos, nem alterações cerebelares ou de pares cranianos. A Ressonância Magnética não detectou qualquer alteração, mas o SPECT cerebral indicou hipoperfusão em regiões parietais e temporais.

\section{Avaliação neuropsicológica}

Ao ingressar no tratamento, FS foi submetido a avaliação neuropsicológica visando traçar um perfil de funcionamento cognitivo para que se pudesse programar a estratégia de intervenção clínica. A quantificação das dificuldades de memória foi feita através do Teste Comportamental de Memória de Rivermead (TCMR) ${ }^{12}$. Este é um teste ecológico que avalia habilidades requeridas em tarefas mais semelhantes às da vida cotidiana. Por ter sido elaborado a partir de questionários para registro de problemas de memória no dia-a-dia, este teste traz dados mais relevantes à reabilitação do que instrumentos baseados em material experimental, já que a intervenção deve ser voltada a minimizar o impacto das dificuldades na vida real do paciente ${ }^{13}$. Ao final do tratamento, 6 meses após, foi realizada uma retestagem, utilizando-se o mesmo instrumento.

\section{Treinamento no computador}

O objetivo do treinamento era ensinar FS a operar um editor de texto (Word for Windows), confeccionando papéis personalizados (receituários, anotações, cartas etc) e cartões de visitas.

O treinamento todo teve duração de 14 semanas, com frequência média de 3 sessões semanais de 50 minutos de duração. FS ficava em uma sala, trabalhando diretamente no computador, e era acompanhado pela neuropsicóloga que o ensinava e registrava seu desempenho.

As técnicas de ensino utilizadas foram a prática repetitiva e a aprendizagem sem erros (errorless learning). A prática repetitiva consiste na execução sucessiva de uma tarefa, obedecendo a etapas pré-estabelecidas e utilizando-se regras constantes. A técnica de aprendizagem sem erros procura contornar a persistência dos erros observada em pacientes amnésticos. Devido às dificuldades de memória explícita, estes pacientes não têm acesso às suas tentativas prévias em que as respostas incorretas foram corrigidas e, desta forma, os erros tornam-se itens competitivos difíceis de serem suprimidos no processo de aprendizagem. Assim, a aprendizagem sem erros consiste em estruturar a situação de treinamento e fornecer o máximo de pistas necessárias para que o paciente não cometa erros, fortalecendo a emissão de respostas $\operatorname{certas}^{9,10}$.

As tarefas ensinadas no computador foram divididas em dois tipos de procedimentos: 1) básicos e 2) específicos. Os procedimentos básicos eram os necessários a qualquer atividade no editor de textos, e foram treinados através de cópia de pequenos textos, além de outros exercícios; os procedimentos específicos eram aqueles que possibilitavam concluir o papel personalizado ou cartão de visitas (Tabela 1).

Os procedimentos eram compostos por séries de comandos, também divididos em dois tipos, de acordo com sua complexidade: A) fundamentais e B) adicionais. Os comandos fundamentais eram os primeiros a serem ensinados e sem eles a tarefa não poderia ser cumprida; os comandos adicionais não eram indispensáveis à conclusão da tarefa, mas eram mais refinados e dependiam do domínio de informações adicionais (Tabela 1).

O cronograma de treinamento foi o seguinte: durante as 3 primeiras semanas trabalhou-se apenas com os procedimentos básicos. Depois, outros procedimentos e comandos foram acrescentados, mas os básicos continuaram a ser utilizados durante todo o treinamento. Desde a $4^{\mathrm{a}}$ até o final da $8^{\mathrm{a}}$ semana FS aprendeu e praticou a confecção de papel personalizado. Após uma pausa de duas semanas, voltou a fazer esta tarefa por mais 4 sessões nas quais avaliou-se o seu desempenho. A partir da $8^{\mathrm{a}}$ semana ele iniciou a confecção de cartões de visitas, praticando até o final do treinamento.

\section{RESULTADOS}

Avaliação de memória pré-treinamento - TCMR: Observaram-se dificuldades de memória para recordação livre de nomes e de histórias, reconhecimento de figuras familiares e execução de tarefa previamente combinada (marcar uma consulta); o desempenho foi melhor no reconhecimento de faces desconhecidas, na recordação de atos prévios (pedir de volta seu pertence), na aprendizagem de sequência motora (caminho) e na orientação (Tabela 2).

Avaliação de memória pós-treinamento (TCMR): na segunda testagem o desempenho geral do paciente não variou em relação à primeira avaliação, mas houve oscilações nos dois momentos em várias tarefas, pois FS conseguiu realizar melhor algumas delas, enquanto que em outras ele foi pior (Tabela 2). Nas atividades cotidianas foram observados dados qualitativos que corroboram as oscilações verificadas no teste, como por exemplo o fato de FS lembrar-se ou não do nome das terapeutas ou do número do andar da sala de atendimento. 
Tabela 1. Procedimentos e Comandos Ensinados

\begin{tabular}{ll}
\hline Procedimentos Básicos & Procedimentos específicos \\
\hline Inicialização & Papel Personalizado \\
1. Ligar estabilizador (A) & 1. Nome acima e à esquerda (A) \\
2. Ligar máquina (A) & 2. Profissão na linha abaixo do nome (A) \\
3. Ligar video (A) & 3. Ir ao fim da folha com a tecla Enter (A) \\
4. Inserir disquete (A) & 4. Tentar $1^{\circ}$ setas, depois Enter (B) \\
5. Iniciar Word (A) & 5. Endereço abaixo e à direita (A) \\
6. Iniciar Word via atalho (B) & 6. Telefone abaixo do Endereço (A) \\
7. Abrir novo documento (A) & Cartão de Visitas \\
Formatação de texto & 1. Abrir documento Mestre (A) \\
1. Negrito (A) & 2. Substituir roteiro (A) \\
2. Itálico (A) & 3. Escrever sobre o roteiro (B) \\
3. Sublinhado (A) & 4. Selecionar cartão modelo (A) \\
4. Alinhamento à direita (A) & 5. Copiar cartão selecionado (A) \\
5. Alinhamento centralizado (A) & 6. Colar os demais cartões (A) \\
6. Pular linhas (A) & \\
7. Selecionar texto com mouse (A) & \\
8. Selecionar texto com teclado (B) & \\
9. Trocar tamanho (A) & \\
10. Trocar tamanho via atalho (B) & \\
11. Trocar tipo (A) & \\
12. Trocar tipo via atalho (B) & \\
13. Trocar cor (A) & \\
Salvar & \\
1. Salvar (A) & \\
2. Salvar em disquete (B) &
\end{tabular}

(A) comandos fundamentais; (B) comandos adicionais.

Tabela 2. Avaliação de memória com o TCMR pré e pós treinamento no computador.

\begin{tabular}{lcc}
\hline & $\begin{array}{c}\text { avaliação prél } \\
\text { perfil padronizado* }\end{array}$ & $\begin{array}{c}\text { avaliação pós/ } \\
\text { perfil padronizado* }\end{array}$ \\
\hline Nome e sobrenome & 0 & 0 \\
Pedir de volta seu pertence & 2 & 2 \\
Marcar uma consulta & 0 & 2 \\
Figuras familiares & 0 & 1 \\
História - imediata & 0 & 0 \\
História - tardia & 0 & 1 \\
Faces desconhecidas & 2 & 1 \\
Caminho imediato & 2 & 1 \\
Caminho tardio & 2 & 0 \\
Recado & 1 & 0 \\
Orientação & 2 & 2 \\
Data & 2 & 2 \\
Total & 13 & 12 \\
Nível da Memória** & prejuízo moderado & prejuízo moderado \\
\hline
\end{tabular}

*descrição dos escores: 2 , realizou adequadamente; 1 , realizou parcialmente; 0 , não realizou a tarefa.

** variando entre: normal, memória fraca, prejuízo moderado e prejuízo severo. 


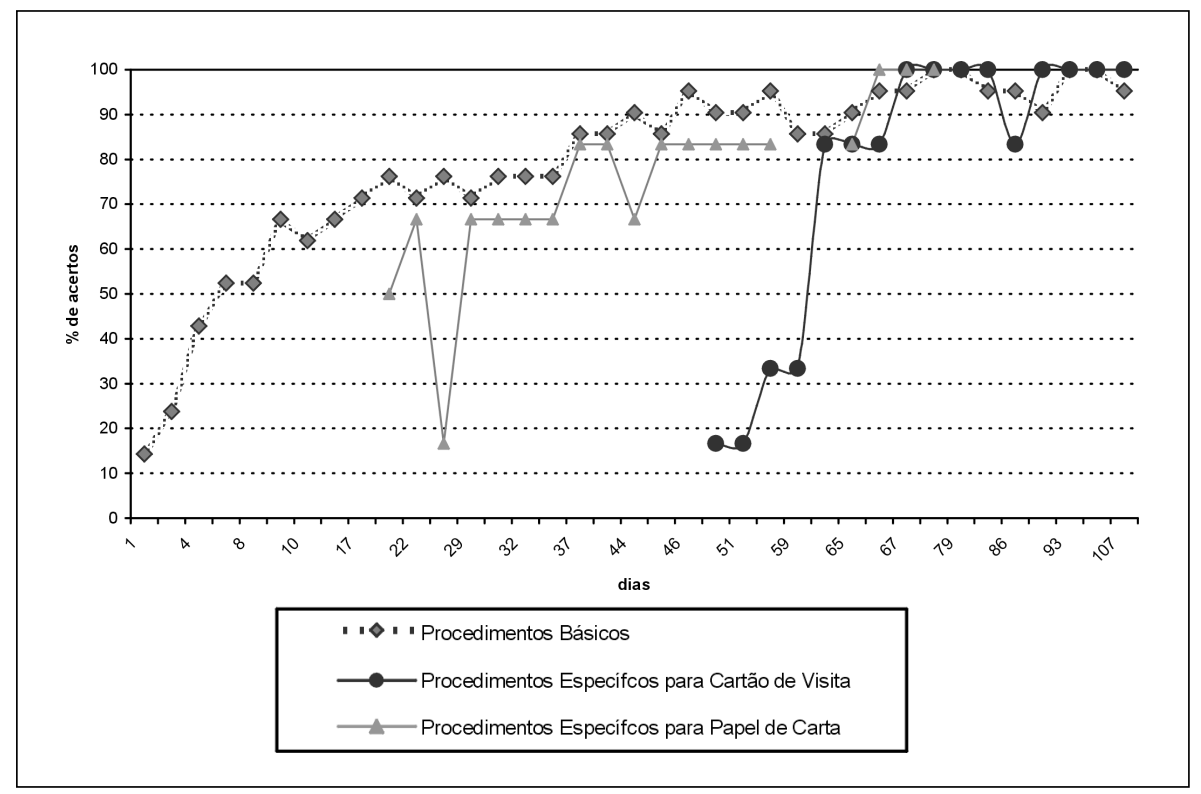

Fig 1. Desempenho nas atividades de computador.

Aprendizado dos comandos: a Figura 1 mostra o desempenho no computador ao longo das 14 semanas. FS apresentou uma curva típica de aprendizagem, com incremento gradual dos acertos, tornando-se capaz de realizar as tarefas propostas (papel personalizado e cartão). As variações diárias em sua pontuação se devem à oscilação no uso de comandos mais complexos.

Além disto, houve situações diferentes, como, por exemplo, um cliente pedir para não colocar a profissão no cartão, ou pedir o nome todo em maiúsculas. Ocorreu também de ele esquecer algum passo. Nesses momentos, FS encontrava dificuldades para concluir a tarefa, sendo que às vezes era necessário retomar o processo a partir de um dos passos anteriores ou mesmo desde o início.

\section{Evolução}

Ao final das 14 semanas de treinamento no computador, FS estava completando 6 meses de atendimento no Centro. Além deste treinamento específico, também participava de sessões individuais de psicoterapia e aprendizagem de estratégias compensatórias para memória. Havia aprendido a utilizar dois tipos de auxílios externos (agenda e caderno de memória), extraindo benefícios destes em sua vida diária.

Durante o aprendizado no computador ficava muito satisfeito em verificar sua melhora e sua produção. Vendeu alguns trabalhos para conhecidos e oferecia seus serviços em lugares que frequentava (barbeiro, sala de espera do Centro, etc).

Os teste formais de memória, por outro lado, não mostraram recuperação específica nesta área.

\section{DISCUSSÃO}

Os resultados obtidos no processo de treinamento de computador mostraram que o paciente teve grande evolução no seu desempenho ao longo das semanas, conseguindo realizar as duas tarefas (papel personalizado e cartão) de forma satisfatória.

Primeiramente, é importante ressaltar que a aprendizagem de tarefas complexas em pacientes amnésticos não depende exclusivamente de um único sistema de memória, refletindo tanto 
aprendizagem explícita como implícita ${ }^{10}$. O paciente FS, especificamente, demonstrava em muitos momentos que era capaz de reter algum material declarativo, por exemplo, lembrando-se do assunto geral de uma conversa de dias anteriores. Desta forma, o seu sucesso no treinamento não deve ser produto exclusivamente de aprendizagem implícita, mas sim de uma interação de processos mnésticos operando na aquisição de conhecimentos, apoiados em estratégias de aprendizagem implícita e de memória explícita residual.

Entretanto, a hipótese de que os processos subjacentes à sua aprendizagem do uso de computador apoiam-se em grande medida em mecanismos de memória implícita pode ser reforçada pelas características de FS durante o seu treinamento, pois ele não era capaz de lembrar-se da situação de treino a não ser vagamente, embora seu progresso diário na aquisição dos comandos estivesse documentado. Além disto, o paciente não era capaz de ter clareza sobre sua evolução, pois não conseguia lembrar-se do seu desempenho no dia anterior. A forma de ajudá-lo a perceber sua melhora no desempenho era fazer com que ele marcasse seus pontos em uma planilha em que os dias anteriores já estavam registrados.

A aprendizagem implícita é caracterizada por ser rígida e hiperespecífica, não permitindo ao paciente fazer um uso flexível do que foi aprendido ${ }^{2}$. Assim, pacientes amnésticos não conseguem transpor o conhecimento adquirido para contextos diferentes. Isto pode ser observado na dificuldade que FS demonstrava para lidar com situações novas ou com pequenas alterações no contexto de trabalho no computador. Além desta dificuldade, o fato de a segunda avaliação da memória não haver mostrado alterações frente aos resultados obtidos previamente ao treinamento, mostra que exercícios de memória em tarefas específicas (no caso, o procedimento no computador) não garantem melhora do funcionamento da memória como um todo, o que está de acordo com muitos relatos anteriores ${ }^{13-15}$.

Glisky e Schacter ${ }^{6}$ sugerem, então, que a reabilitação de pacientes com problemas de memória deve ser focada no ensino de conhecimento específico (domain-specific knowledge), ou seja, em informações, tarefas e habilidades que possam ser diretamente aplicadas em um âmbito particular da vida diária do paciente.

Além de ser contextualizado e específico, o presente treinamento teve outras características que certamente facilitaram o sucesso de FS: cada tarefa podia ser dividida em passos menores e mais simples e, uma vez aprendidas, as tarefas poderiam ser executadas de forma repetitiva e sem alterações que demandassem flexibilidade do conhecimento. Segundo Glisky, ${ }^{8}$ é fundamental observar que fatores como estes estejam assegurados nas tarefas a serem treinadas com pacientes amnésticos.

Dentre as dificuldades encontradas, FS mostrou que mesmo as menores alterações exigidas na tarefa podiam dificultar seu desempenho. Entretanto, com a passagem do tempo e a solidificação do conhecimento adquirido, o paciente conseguia realizar incrementos ou pequenas alterações nas tarefas, concluindo-as de forma satisfatória.

Estes dados condizem com os resultados de Glisky ${ }^{8}$, que observa que informações bem aprendidas podem suportar pequenas alterações, especialmente se isto for processado de maneira implícita, isto é, se as modificações requeridas puderem ser solicitadas dentro da própria tarefa.

Desta forma, a técnica de aprendizagem sem erro mostrou-se adequada ao trabalho com este paciente amnéstico, facilitando a solidificação das respostas corretas, além de ser extremamente eficiente na manutenção do engajamento e motivação, já que com esta técnica o paciente sempre era bem sucedido nas tarefas realizadas ao longo do treinamento.

Agradecimentos - Ao paciente FS e sua família pela assiduidade e disposição, sem os quais seria impossível a realização deste trabalho. A Juliana Lemos Garcia Ferreira e Alexandra Britto Bezerra pela importante participação na elaboração da metodologia de trabalho. À Associação Fundo de Incentivo à Psicofarmacologia que oferece as instalações e o suporte financeiro para o desenvolvimento dos trabalhos do Centro Paulista de Neuropsicologia. 


\section{REFERÊNCIAS}

1. Schacter DL. Implicit memory: history and current status. J Exp Psychol Learn Mem Cogn 1987;13:501-518.

2. Squire LR. Memory and brain. New York: Oxford Univ Press, 1987.

3. Oliveira MGM, Bueno OFA. Neuropsicologia da memória humana. Psicol USP 1993;4:117-138.

4. Graf P, Schacter DL. Implicit and explicit memory for new associations in normal and amnesic subjects. J Exp Psychol Learn Mem Cogn 1985;11:501-518.

5. Tulving E, Schacter DL, Stark H. Priming effects in word-fragment completion are independent of recognition memory. J Exp Psychol Hum Learn Mem 1982;8:336-342.

6. Glisky EL, Schacter DL. Extending the limits of complex learning in organic amnesia: computer training in a vocational domain. Neuropsychologia 1989;27:107-120.

7. Wilson BA, Baddeley AD, Cockburn JM. How do old dogs learn new tricks: teaching a technological skill to brain injured people. Cortex 1989;25:115-119.

8. Glisky EL. Acquisition and tranFSer of declarative and procedural knowledge by memory-impaired patients: a computer data-entry task. Neuropsychologia 1992;30:899-910.

9. Baddeley AD. Implicit memory and errorless learning: a link between cognitive theory and neuropsychological rehabilitation? In: Squire LR. Neuropsychology of Memory, 2.Ed. New York: Gilford Press, 1992.

10. Wilson BA, Baddeley AD. When implicit learning fails: amnesia and the problem of error elimination. Neuropsychologia 1994;32:53-68.

11. Glisky EL, Schacter DL, Tulving E. Computer learning by memory-impaired patients: acquisition and retention of complex knowledge. Neuropsychologia 1986;24:313-328.

12. Wilson BA, Baddeley AD, Cockburn JM. The Rivermead Behavioural Memory Test. 2.Ed. Tradução Oliveira RM, Schmidt SL. Suffolk: Thames Valley Company, 1991.

13. Wilson BA. Cognitive rehabilitation: how it is and how it might be. J Int Neuropsychol Soc 1997;3:487-496.

14. Prigatano GP, Glisky EL, Klonoff PS. Cognitive rehabilitation after traumatic brain injury. In: Corrigan PW, Yudofsky SC. Cognitive rehabilitation for neuropsychiatric disorders, Washington, DC: American Psychiatric Assoc Press, 1996.

15. Ericson KA, Chase GE, Falcon S. Acquisition of a memory skill. Science 1980;208:1181-1182. 